\title{
Anesthesia Equipment: Principles and Applications (Second Edition)
}

\author{
Jan Ehrenwerth, James B. Eisenkraft, James M. Berry. ExpertConsult.com. \\ Elsevier Saunders, Philadelphia, 2013. ISBN 978-0-323-11237-6
}

\author{
Kenneth M. LeDez, MBChB
}

Received: 26 June 2013/Accepted: 15 July 2013/Published online: 30 July 2013

(C) Canadian Anesthesiologists' Society 2013

It is a troubling reality that much of the technology used by anesthesiologists every day is all too often taken for granted and little understood. The pressures of residency training and clinical practice are such that equipment basics may be neglected. Does Anesthesia Equipment: Principles and Applications help to fill that gap? With so much technological development, how can a book on equipment remain up to date? Is there anything in the book that a resident in training or a staff anesthesiologist would not learn in practice? Is this a book that would simply look good on a shelf or coffee table but would not actually be used or read?

This book is well-written and readable, not an easy task for a book on equipment. Furthermore, the quality and clarity of the many colour illustrations and photographs are impressive, for example, the detailed illustrations and photographs of anesthesia vaporizers are superb and could be useful even for very experienced anesthesiologists.

The overall structure and organization of the book may not please everyone. For instance, although Part II is titled SYSTEM MONITORS and Part III is titled PATIENT MONITORS, the reasons for this separation are not apparent as much of Part II deals with respiratory flow, pressure, and gas monitoring of patients. The "Machine Checkout" is located in Part VII SAFETY, STANDARDS, AND QUALITY, which is near the end of the book rather than near the beginning, and the chapter on "Airway Equipment" is located in Part IV OTHER EQUIPMENT. Given the central importance of airway management in anesthesiology, some may find this placement less than ideal. Nevertheless, the content of all components of this

K. M. LeDez, MBChB ( $\square)$

Memorial University, St. John's, NL, Canada

e-mail:kledez@mun.ca book are well researched and presented in an organized and logical manner. Chapter 18 "Infusion Pumps" is one example of many sections in this book that are concise, well structured, and contain a wealth of excellent illustrations and data.

What is missing or omitted from Anesthesia Equipment: Principles and Applications? There is no mention of bispectral index or other neurologic monitoring methods, such as evoked potentials, cerebral oximetry, or transcranial Doppler. Other uses of Doppler ultrasound are also absent. Indeed, the book omits transesophageal echocardiography and largely also ultrasound for vascular access and nerve block, aside from the section about simulation. The topic of nerve stimulators is covered but only for monitoring neuromuscular blockade and not for use in regional anesthesia. There is extensive coverage of video laryngoscopes, but no pictures of the various direct laryngoscope blades that are described in Box 16-1. Sleep apnea seems to have reached almost epidemic proportions among patients presenting for anesthesia; however, continuous positive airway pressure and various other forms of ventilatory support equipment that are commonly used in recovery rooms are not included in the book. The word "Venturi" does not appear in the index, oxygen masks are not discussed, and nasal cannulae are mentioned only in the section on fire safety, yet these are everyday items used during regional anesthesia, sedation, and in the recovery room. That being said, the book contains a wide breadth of information; the text and illustrations on electrical and fire safety are among many examples that are well covered in this publication.

There are some important strengths of this book. The expertise of the authors of the chapter on "Standards and Regulatory Considerations" is evident, as this may be the best concise text available on this topic. Another excellent chapter is "The Hazards of the Anesthesia Delivery 
System". The comprehensive chapters dealing with informatics, computers, alarms, ergonomics, vigilance, and systems integration demonstrates the success of this book as being up to date and relevant for current and future anesthesiology practice. The use of simulation receives extensive attention as does electronic charting and anesthesia information management systems. The inclusion of a very detailed chapter on infection prevention in an equipment text is commendable. It might be argued by some that the effect is spoiled a little by a photo of handwashing at a sink not intended for an aseptic scrub (Fig. 20-1) and by another photo showing a practitioner wearing a mask but not using a sterile drape and gown while performing a neuraxial technique (Fig. 20-5). Overall, however, the inclusion of many visual illustrations of important information facilitates easy reading.

The intended readership appears to be mainly American, e.g., the abbreviation "CSA" is used for "compressed spectral array" rather than for Canadian Standards Association. Nevertheless, the distinctions between Canadian and American practices are well covered in the section on medical gases. Elsewhere in the book, there is considerable mention of European and international standards.

Purchasers receive an activation code that gives Internet access to online searchable materials for this book, but this feature was not available to the reviewer to assess. Anesthesia Equipment: Principles and Applications does not present everything on the subject, but it offers a great deal. It is not flawless, and improvements can certainly be made in future editions, but it is a good book and a worthwhile purchase that will likely be used rather than simply look good on a shelf.

Conflicts of interest None declared. 